\title{
Las dos últimas décadas en el estudio de la conformidad y la influencia social
}

\section{José Miguel Fernández Dols}

Universidad Autónoma de Madrid

¿Cómo deben entenderse aquellas situaciones en las que un grupo puede hacernos modificar nuestros juicios haciendo incluso que incurramos en error? Para responder a tal pregunta, Asch (1951, 1952) parte de dos presupuestos claves: la independencia cognoscitiva del sujeto y la concepción de que la cognición social del individuo depende de un contexto que le permite establecer un patrón de validación de la realidad.

Los estudios empíricos de Asch sobre el tema son bien conocidos: siete o nueve individuos deben indicar, en una serie de tareas perceptivas bastante sencillas y claras, cuál de varias líneas es igual a otra patrón. Como la tarea no tiene dificultades, todos los sujetos están de acuerdo hasta que, de pronto, se produce un brusco desacuerdo entre el grupo -que establece un determinado juicioy uno de sus miembros que disiente. El sujeto disidente se halla, súbitamente, en una situación incómoda e incomprensible para él, ya que el juicio parece estar claro; le quedan dos alternativas: aceptar el juicio de la mayoría o mostrar su disidencia en esta ocasión y en otras en que pueda ocurrir.
La manipulación clave de esta situación experimental radica, por supuesto, en el hecho de que todos los sujetos excepto uno - situado en los últimos lugares- están de acuerdo con el experimentador para, en determinados ensayos, emitir sin titubeos juicios unánimemente erróneos.

Los resultados del experimento descrito parecen comprobar que, efectivamente, existe una independencia cognitiva del sujeto en la elaboración de sus juicios pero sólo hasta cierto punto: las estimaciones erróneas de la mayoría provocan juicios erróneos en un tercio de las declaraciones de la minoría, aunque existían marcadas diferencias individuales.

Es importante tener en cuenta que, para Asch, la clave de tales muestras de conformidad no radican sino en una serie de características que configuran lo que Sherif (1936) denominó como «marco de referencia»: un conjunto de condiciones que determina el campo perceptivo del sujeto.

El efecto producido por Asch es dramático e implica la imposición de la conducta de un modelo (grupo) a un 
sujeto, proceso unidireccional igualmente atractivo para los teóricos del Aprendizaje Social y para los de la Teoría del Campo. De hecho, Asch se preocupa ante todo por subrayar las causas de la independencia del individuo en el grupo, pero cuando se produce conformidad en contra de lo que él mismo esperaba (Deutsch y Krauss, 1965; Moscovici, 1980) se produce un vacío explicativo que da juego -especialmente durante los años cincuenta- a la aplicación del método experimental de Asch desde visiones teóricas distintas e incluso sin visión teórica alguna.

El planteamiento de Asch se constituye en referencia operacional común para el estudio de la influencia. Como indica Eiser (1980), fueron los experimentos de Asch los que establecieron el término «conformidad» como un elemento importante del repertorio explicativo del psicólogo social.

\section{LOS AÑOS CINCUENTA}

A pesar del éxito del procedimiento experimental, los psicólogos sociales prestaron poca atención al modelo explicativo del autor (Allen y Wilder, 1980), que fue reinterpretado habitualmente con connotaciones que recuerdan, a menudo, los planteamientos de la escuela lewiniana y el Grupo de Yale.

Blake y Mouton realizan en 1961 una consideración global de los resultados de la investigación sobre conformidad, fundamentalmente durante la década de los cincuenta.

Es interesante observar cómo, según Blake y Mouton, son tres las características del material que elicitarían una mayor conformidad: la dificultad de los ítems, la carencia de indicios de validación ajenos a la propia conducta de los miembros del grupo y la presión hacia la conformidad a las instrucciones.

Los investigadores de esta década conciben el marco de referencia como algo que se elabora para la interpretación de un fenómeno, sin que el propio fenó- meno forme parte de la elaboración misma del marco de referencia. No se trata de observar si el sujeto elabora un patrón perceptivo a partir de una determinada información, sino de comprobar si emite una respuesta «correcta o incorrecta ante un ítem por medio de unos criterios de objetividad ya establecidos». La unidireccionalidad del proceso es evidente. La situación de conformidad pierde su carácter de actividad cognitiva en términos de estructuración del medio y se convierte en una actividad meramente reactiva que acentúa un fenómeno ajeno a los planteamientos iniciales de Sherif y Asch.

Ante tal panorama el propio Asch indica en 1961 que:

«Los efectos seleccionados para su estudio generalmente han implicado poca sensibilidad hacia las condiciones percibidas. Parecen mostrar que uno puede manipular el juicio arbitrariamente, sean cuales sean las circunstancias dadas. La principal condición que los psicólogos han extraído de esta región es que uno puede inducir a las personas a creer lo que los otros sin más razón que la de que los otros lo creen...» (Asch, 1961, pp. 150-151).

Al comienzo de los años sesenta se siente la necesidad de un estudio de los procesos de conformidad que integre una relación bidireccional entre el sujeto y el grupo. Sin embargo, para comprender cuáles van a ser las principales líneas de investigación durante la década de los sesenta es preciso tener en cuenta algunas otras aportaciones importantes que matizan o perfeccionan los aspectos heurísticos o teóricos del estudio de la conformidad. Se trata de los trabajos de Deutsch y Gerard (1955) y Thibaut y Kelley (1959).

El trabajo de Deutsch y Gerard supone una matización a los estudios lewinianos sobre las fuerzas que rigen la interdependencia grupal. Para estos autores existen dos tipos de influencia social en experimentos como el de Sherif o el de Asch. La influencia social normativa es «la influencia a conformarse a las expectativas positivas del otro»; «expec- 
tativas positivas» son aquellas cuyo cumplimiento «lleva o refuerza sentimientos positivos más que negativos». La influencia social informativa es más bien la influencia «para aceptar la información obtenida del otro como evidencia sobre la realidad». Aunque ambos fenómenos suelen, según Deutsch y Gerard, presentarse entrelazados, es posible conformarse sin creer en lo que se hace y «aceptar las creencias del oponente como evidencias de la realidad aun cuando no se esté motivado a coincidir con él».

Deutsch y Gerard redescubren una observación de Asch: la influencia normativa no es la única en la situación de conformidad, existe la influencia incluso si el sujeto no se halla normativamente influenciado.

Sin embargo, tal puntualización va a tener, sobre todo, importancia en la década de los sesenta, a partir quizá de su difusión generalizada en el manual de Jones y Gerard (1967) y, sobre todo, de la atención creciente que se presta al aspecto cognitivo de la influencia social a partir de la Teoría de la Atribución (Heider, 1958).

Más significativa de la actividad de esta década es la confluencia de John W. Thibaut y Harold H. Kelley en la Teoría del Intercambio (1), con su obra The Social Psychology of Groups (1959). Según la versión de la Teoría del Intercambio a cargo de Thibaut y Kelley, los interactuantes procuran «negociar» con el otro un patrón de conducta lo más beneficioso posible. La influencia social descansa en un adecuado control de la conducta en términos de una economía de refuerzos, economía en la que deben participar voluntariamente - por interés compartido- el sujeto influyente y el influido:

«El problema de $B$ es reforzar la tendencia de A a actuar del modo deseado sin hacer demasiado visible su dependencia respecto a A. Esto se hace apelando a un valor supraindividual (...); las normas se basan en un acuerdo o consenso que reduce la necesidad de vigilancia (...) (y) reducen el grado en que la sumisión se percibe co- mo una cuestión de ceder ante una persona más poderosa.»

La Teoría del Intercambio va a plantear uno de los modos de interpretación de la situación de influencia durante los años sesenta (Nord, 1969) y posee, para el argumento del presente escrito, dos aportaciones de interés: restablece la relación bidireccional en el mantenimiento del orden social y tiene en cuenta la actividad cognitiva de los sujetos, aun cuando ésta sea solamente un supuesto ineludible para la comprensión de la relación de intercambio y no un objeto real de estudio en las investigaciones empíricas de estos autores (Morales, 1981).

\section{LA INVESTIGACION EN LOS AÑOS SESENTA}

La Teoría del Intercambio fue uno de los productos de la década de los cincuenta que, durante los años sesenta, va a tener su momento álgido, ya que coinciden en la tarea los psicólogos, por su propia dinámica de estudio, y los sociólogos, en los que:

«... su rechazo del modelo consensual (...) lleva a reconsiderar algunas de las cuestiones clásicas fundamentales de la teoría social. Por ejemplo, la reconsideración del «problema hobbesiano del orden» en el contexto de una renovada teoría utilitaria (es decir, del intercambio)» (Buckley, 1970, pp. 205-206).

Además, el modelo de intercambio recibe un complemento teórico a partir de la Teoría de la Atribución (Heider, 1958). El propio Harold H. Kelley (1967), uno de los padres de la Teoría del Intercambio indica que:

«En nuestro libro The Social Psychology of Groups Thibaut y yo hemos hecho un análisis de la dependencia (e interdependencia) en términos de resultados -outcomes- (recompensas y costos). Dicho análisis supone que tendremos, en último término, la capacidad suficiente para medir o establecer un índice del nivel de resultados (outcomes) de una persona. Propone- 
mos ahora que se haga un análisis similar de la dependencia informacional ( $y$ la interdependencia). Y suponemos que en último término los psicólogos proporcionarán un índice significativo del nivel de información de la persona a lo largo de las líneas generales sugeridas anteriormente» (Kelley, 1967, pp. 198-199).

En el caso de la dependencia y la influencia informacional, la pregunta clave para Kelley sería: «cuando una persona tiene la impresión de que algo respecto a una entidad es verdad, ¿cómo determina que su impresión refleja las propiedades inherentes de la entidad y no sus propias características o alguna interacción peculiar con la entidad?»

La atribución del sujeto depende de la información que obtiene. La dependencia e influencia queda definida objetivamente por los efectos de la información del sujeto influyente sobre el influido $\mathrm{y}$, en términos subjetivos, por la creencia - por parte del sujeto dependiente- de que el sujeto influyente va a proporcionarle el mejor nivel de información posible.

Toda situación que implique inestabilidad en la atribución del sujeto favorecerá la conducta conformista que no es sino una búsqueda de conductas consistentes o consensuadas. Por lo demás, las características que proporcionan validez a un fenómeno son las mismas que la proporcionan a un sujeto influyente (constancia, consenso, univocidad, etc.). Los criterios de atribución de validez describen «las distintas fuentes informativas que una persona puede tener para una creencia».

Como se ve, la Teoría de la Atribución pretende salvar la dicotomía, no superada en la Teoría del Intercambio, entre realidad objetiva -plano interactivo, conductual - y el plano referencial, subjetivo, de los procesos de influencia social. Mediante una serie de bipótesis normativas supone cuál es la conducta característica del sujeto que se conforma a nivel informacional, frente al sujeto que se conforma a nivel estrictamente conductual.
En realidad, tanto la Teoría del Intercambio como la Teoría de la Atribución se configuran en términos de hipótesis normativas que han dificultado el desarrollo empírico de los estudios sobre procesos de conformidad. Utilizo el término «hipótesis normativas» en el sentido de que no se estudian directamente los procesos cognitivos y conductuales implicados, sino unas «normas generales de actuación» que solamente se infieren indirectamente a partir de la conducta de los sujetos; de hecho, no se supera, estrictamente hablando, el nivel de descripción de conductas aunque se infiere a partir de él una serie de «reglas de conducta» no regidas estrictamente por los principios de la teoría.

En el caso de la Teoría del Intercambio, Morales (1981) indica que:

«... ofrece una serie de explicaciones plausibles sobre los fenómenos más importantes de la interacción (...); sin embargo, no se la puede considerar como una teoría en el sentido estricto de la palabra (..): los conceptos fundamentales no se han operacionalizado» (Morales, 1981, p. 220).

Y, en el caso de la Teoría de la Atribución, Eiser (1980) indica que:

«... hemos llegado a una etapa en la que ya no es útil, si es que lo ha sido alguna vez, poner a prueba y "verificar" la teoría de la atribución (...). Esto no supone afirmar que no se pueden derivar predicciones de una u otra versión teórica y someterlas a comprobación empírica, sino que la contribución más útil de la teoría de la atribución ha sido proporcionar un marco heurístico de trabajo para definir los procesos cognitivos implicados en la percepción interpersonal. La investigación empírica debería fijar su meta en la identificación de los factores situacionales e individuales que afectan a tales factores» (Eiser, 1980, página 124).

Por lo demás, la necesidad de una consideración en términos de procesos cognitivos y conductuales fue, en la década de los sesenta, una exigencia de diversos autores que critican las deficiencias conceptuales del estudio de la conformidad y los procesos de influencia 
social (Jahoda, 1959; Kelman, 1958, 1961; Willis, 1965; Hollander, 1965; Hollander y Willis, 1967).

En este marco, dos autores elaboran nuevas perspectivas en la consideración del problema: Stanley Milgram y Serge Moscovici. Ambos publican sus trabajos más conocidos en la década de los sesenta (Milgram, 1963, 1964, 1965) (Moscovici et al, 1969), pero su influencia se va a extender hasta la siguiente década con la publicación de sus trabajos anteriores en dos libros: Obedience to $\mathrm{Au}$ thority (Milgram, 1974) y Social Influence and Social Change (Moscovici, 1976), ambos traducidos al castellano.

\section{EL ESTUDIO \\ DE LA CONFORMIDAD \\ DURANTE LOS ULTIMOS DIEZ AÑOS}

Los experimentos de Milgram sobre obediencia a la autoridad son, quizá, uno de los planteamientos experimentales más controvertidos de la última década (vid., p. ej., Miller, 1972). Como se sabe, se compararon las predicciones de psiquiatras y estudiantes y adultos de clase media con los resultados reales de una situación en la que se ordenaba a un sujeto, en un supuesto ejercicio de memoria, aplicar descargas a otro en un rango que comenzaba con 15 voltios y terminaba con 450 voltios.

Resultó impresionante para la opinión pública que, mientras la media predicha fue que los administradores de descargas se detendrían a los 135 voltios, la realidad fuera otra: los sujetos, a pesar de oír las quejas de la víctima, siguieron administrándole descargas con unos valores medios que oscilaron (según la proximidad de la víctima) entre 270 y 405 voltios.

Existieron otras variaciones del diseño original, pero lo que aquí nos interesa no son las implicaciones metodológicas o éticas que implica el experimento de Milgram, cuanto que supone un aldabonazo para la Teoría de Intercambio y la Teo- ría de la Atribución y, en general, para todos los estudios que subrayan la independencia y utilización óptima de alternativas por parte de los sujetos en situación de influencia.

La experiencia de Milgram demuestra que una cosa es tener en cuenta los aspectos cognitivos y conductuales de la situación de influencia, como en su momento los tuvo Sherif, y otra muy distinta suponer que el sujeto es una entidad perfectamente diferenciada; un ente pensante que estudia sus posibilidades y decide, en consecuencia, tomando perspectivas respecto a su entorno.

El sujeto concentra su atención en la autoridad, reinterpreta la situación en términos de la explicación implícita o explícita de la autoridad, etc. El sujeto entra en una dinámica conductual que desdibuja la relevancia del «sujeto calculador» de la Teoría del Intercambio y la Teoría de la Atribución:

«Algunas personas interpretan la situación experimental como que el sujeto, de un modo latamente racional, puede superar los valores en conflicto de la situación, procesar los factores de acuerdo con algún cálculo mental, y basar su acción en el producto de tal ecuación (...). Este análisis ignora un aspecto crucial de la conducta iluminado por los experimentos. Aunque muchos sujetos tomen intelectualmente la decisión de que no deberían administrar más descargas a las víctimas son frecuentemente incapaces de transformar su convicción en acción» (Milgram, 1974, p. 148).

La obra de Milgram ha sido, sin duda, una influencia estimulante en el estudio de la conformidad, pero no ha generado una línea de investigación explícita.

Los trabajos de Moscovici, sin embargo, sí han creado escuela en el seno de la Psicología Social europea. En la introducción de su obra más relevante (Social Influence and Social Change), Moscovici parte de un supuesto discutible: el tratamiento del tema de la influencia social se ajustaría a un modelo único - «funcionalista»- que, al parecer, ha uniformado su estudio. Tal modelo acentuaría la visión del sujeto como pasivo, 
dependiente y reactivo, mientras que Moscovici propone una nueva perspectiva que considera al medio social como producto de la interdependencia y la interacción de los individuos, y no como algo dado ante lo cual el sujeto reacciona. Tal nueva perspectiva, consonante con los principios de la Teoría del Intercambio de autores como Thibaut y Kelley (inter- acción como lucha y negociación y observación de los productos de tal interdependencia) es denominada «modelo genético».

En resumen, la conclusiớn fundamental de Moscovici será que cualquier grupo o individuo puede ser objeto de influencias, sea mayoría o minoría. Las minorías pueden ejercer influencia y algunos de sus experimentos (Moscovici et al., 1969) parecen demostrarlo: cuando una mayoría se enfrenta a una minoría que se mantiene consistente en sus juicios en una tarea de percepción de colores (verde o azul) sufre un «desplazamiento» hacia el color postulado por la minoría en situaciones posteriores de juicio perceptivo; ha habido una influencia larvada de la minoría.

La razón de que la influencia pueda ser ejercida por la minoría radica en que la clave de la influencia consiste en pro ducir un conflicto. Planteando de forma ambigua la distinción entre conflicto social y conflicto conceptual (Berlyne, 1960), Moscovici considera que la disconformidad produce, ante todo, conflictos ansiógenos que los sujetos tratan de reducir no cognitiva sino socialmente, tratando de converger hacia la postura conflictiva. La incertidumbre se deriva, pues, del conflicto, y la influencia es un modo de negociación de tal conflicto. En tal negociación la clave del éxito radica en los «estilos conductuales»: el individuo implicado, autónomo y, sobre todo, consistente pero no dogmático es un individuo influyente.

Nótese que Moscovici plantea una visión del individuo influyente (minotía influyente) que deriva hacia el tema del cambio social y donde, en último término, se subrayan exageradamente al- gunos aspectos de la Teoría del Intercambio: negociación como base de la influencia para la resolución de un conflicto; interdependencia de las partes implicadas -que se persuaden recíprocamente-y sujetos más o menos conscientemente calculadores.

El trabajo de Moscovici plantea muchos problemas; uno de los más importantes es que la afirmación de que una minoría consistente es influyente resulta experimentalmente discutible. El bloqueo que incita una postura fija de la minoría puede provocar en el grupo polarizaciones aún más radicalizadas, de modo que los autores que han desarrollado la tesis de Moscovici tuvieron que idear la hipótesis subsidiaria de la «flexibilidad» negociadora: son influyentes las minorías consistentes pero «flexibles», quedando tal flexibilidad definida de forma muy imprecisa y circunstancial (vid., p. ej., Mugny, 1980a).

Sin embargo, es indudable que Moscovici ha animado la polémica en torno al problema de la conformidad y es el prototipo de la tendencia actual a considerar la influencia social desde una visión que subraya las estrategias individuales de la conducta conformista e inconformista.

Es interesante constatar cómo la investigación actual sobre los procesos de influencia y conformidad puede entenderse contrastando los contenidos de la Psicología Social europea y norteamericana.

Por una parte, la investigación norteamericana ha concentrado gran parte de sus esfuerzos en la definición de los determinantes cognitivos del proceso de conformidad. Los puntos de referencia en tal tarea son, principalmente, dos: la reconsideración del paradigma explicativo de Asch (vid. Allen y Wilder, 1980, en este mismo dossier) o el desarrollo de la teoría de la atribución (vid. Ross et al., 1976, en este mismo dossier).

En los dos casos los resultados no dan más de lo que prometen (una reconsideración más documentada y matizada de lo que el propio Asch ya afirma o, en los desarrollos del principio de atribu- 
ción, una descripción normativa basada en inferencias indirectas), pero se intuye claramente una búsqueda de modelos explicativos alternativos, un mayor cuidado en los niveles de descripción del fenómeno (Wilder, 1977; Allen, 1980) y la utilización de experimentación en nuevos contextos (Nemeth, 1980; Latané, 1981) y situaciones (Nemeth et al., 1974; Allen, 1975).

En el área europea, diversos autores (Deconchy, 1980; Mugny, 1980, 1981; Personnaz, 1979, etc.), además del propio Moscovici, recalcan la importancia del conflicto y los contextos ideológicos en la situación de influencia. Desde el punto de vista cognitivo importan especialmente fenómenos tales como la categorización ideológica del bando propio y el bando adversario. El proceso de conformidad queda explicado desde diversas versiones del modelo de Moscovici que matizan sus hallazgos experimentales y los encuadran en una perspectiva crítica de tipo político-social.

La perspectiva europea es quizá la más conocida actualmente en España a partir de una serie de traducciones recientes (Moscovici, 1981; Mugny, 1980b; Mugny, 1981; Mugny y Papastomou, 1980a y 1980b), por lo que no aparecerá representada directamente en este dossier.

Sí aparece en el dossier, sin embargo, un artículo de Nemeth (Nemeth et al., 1974; Nemeth y Wachtler, 1974; Nemeth et al., 1977; Nemeth, 1980); una investigadora norteamericana que participa también en la corriente europea (Moscovici y Nemeth, 1974) y que se plantea el estudio de la influencia en términos de negociación, persuasión y decisión grupal. Es significativo que Nemeth encuentre en la negociación que precede a una toma de decisiones (p. ej., en un jurado simulado) el planteamiento básico para el estudio de la influencia pública y privada.

Por lo demás, su trabajo reciente (Nemeth, 1982, vid. dossier) es un buen ejemplo de imaginación en la utilización de diseños alternativos e ilustra las dificultades que encuentran los investigadores para contrastar empíricamente la variable «estilo conductual», clave en el modelo teórico de Moscovici: ¿tienen todas las minorías consistentes la suficiente habilidad expresiva para lograr un estilo conductual «flexible»?; si no es así, ¿qué variable media entre consistencia y estilo conductual y cómo evaluarla experimentalmente?

\section{ADDENDA}

El último apartado de este escrito plantea un enfrentamiento entre la Psicología Social europea y norteamericana. Existe, sin embargo, un nexo común: en ambos bandos la atención se centra en las estrategias individuales que cada individuo adopta en la situación a la que se enfrenta, sea negociación del conflicto sea estructuración cognitiva de la situación.

Por lo demás, el postulado de tal enfrentamiento debe considerarse en sus justos términos: se trata de un guión expositivo que, como todo esquema, ignora una realidad mucho más compleja y multiforme en sus opciones tanto por parte americana (vid., p. ej., Gerard, 1980; Latané, 1981), como por parte europea (vid., p. ej., Avermaet y Doms, 1980; Hatcher, 1982; Montmollin, 1977). Tal riqueza certifica la buena salud de la Psicología Social Experimental, gracias o a pesar de la complejidad de los problemas a los que se enfrenta.

\section{Nota}

(1) Para una exposición más amplia de la obra de estos autores vid. Morales, 1981. 


\section{Referencias bibliográficas}

Allen, V. L.: «Social Support for Nonconformity», en Berkowitz, L. (ed.), Advances in Experimental Social Psychology, Nueva York: Academic, 1975.

AlleN, V. L.: «Infra-group, Intra-group and Inter-group: Units, Levels and Meanings in Social Influence», Simposium Internacional sobre Influencia Social, Barcelona, septiembre 1980.

Allen, V. L., y WILDER, D. A.: «Impact of Group Consensus and Social Support on Stimulus Meaning: Mediation of Conformity by Cognitive Restructuring», J. Person. and Soc. Psychol., $1980,39,1116-1124$.

Asch, S. E.: «Effects of Group Pressure upon the Modification and Distortion of Judgement», en Guetzkow, H. (ed.), Groups, Leadership and Men, Pittsburg: Carnegie Press, 1951.

Ascr (1952): Psicología Social, Buenos Aires: Eudeba, 1964.

AsCH, S. E.: «Issues in the Study of Social Influences on Judgment», en Berg, I. A., y Bass, B. M. (eds.), Conformity and Deviation, Nueva York: Harper, 1961.

Berlyne, D. E.: Conflict, Arousal and Curiosity, Nueva York: McGraw Hill, 1960.

Blake, R. R., y Mouton, J. S.: «Conformity Resistence and Conversion», en Berg, I. A., y Bass, B. M. (eds.), Conformity and Deviation, Nueva York: Harper, 1961.

BuckLEY, W. (1967): La sociología y la teoría moderna de los sistemas, Buenos Aires: Amorrortu, 1970.

DeconchY, J. P.: Orthodoxie religieuse et Sciences Humaines, La Haya: Mouton, 1980.

Deutsch, M., y Gerard, H. B.: «A Study of Normative and informational Social Influences upon Individual Judgment", J. of Abn. and Soc. Psycbol., 1955, 51, 629-636.

Deutsch, M., y Krauss, R. M. (1965): Teorías en Psicología Social, Buenos Aires: Paidós, 1975.

EIsER, J. R.: Cognitive Social Psychology, Londres: McGraw Hill, 1980.

HATCHER, J.: «Arousal and Conformity», en Brandstatter H. et al. (eds.), Group Decision Making, Londres: Academic, 1982.

HeIDER, F.: The Psychology of Interpersonal Relations, Nueva York: Wiley, 1958.

Hollander, E. P.: «Independence, Conformity and Civil Liberties: Some Implications from Social Psychological Research», J. of Soc. Issues, 1975, 31, 55-67.

Hollander, E. P., y Willys, R. H.: «Some Current Issues in the Psychology of Conformity and Nonconformity», Psychol. Bull., 1967, 68, 62-76.

JAHODA, M.: «Conformity and Independence», Human Rel., 1959, 12, 99-117.

Jones, E. E., y Gerard, H. B.: Foundations of Social Psychology, Nueva York: Wiley, 1967.

Kelman, $\mathrm{H}$. C.: "Compliance, Identification and Internalization. Three Processes of Attitude Change», J. of Conflict Resol., 1958, 2, 51-60.

Krlman, H. C.: «Processes of Opinion Change», Public Opinion Quart, 1961, 25, 57-78.

Kelley, H. H.: «Attribution Theory in Social Psychology», en Levine, D. (ed)., Nebraska Symposium on Motivation (vol. XV), Lincoln: Univ. of Nebraska Press, 1967.

Latané, B.: «The Psychology of Social Impact», American Psychologist, 1981, 36, 343-356.

Mrlgram, S.: «Behavioral Study of Obedience», $J$. Abn. and Soc. Psycbol., 1963, 67, 371-378.

Milgram, S.: "Group Pressure and Action Against a Person», J. Abn. and Soc. Psychol., 1964, 69, 137-143.

Milgram, S.: «Liberating Effects of Group Pressure», J. of Person. and Soc. Psychol., 1965, $1,127-134$.

MLgRAM, S. (1974): Obediencia a la autoridad: un punto de vista experimental, Bilbao: Desclée de Brouwer, 1980.

Miller, A. (ed.): The Social Psychology of Psychological Research, Nueva York: Free Press, 1972.

MonTmollin, G. DE: L'Influence sociale. Pbénomenes, facteurs et théories, París: P.U.F., 1977.

Morales, J. F.: La conducta social como intercambio, Bilbao: Desclée de Brouwer, 1981.

MoscovicI, S. (1976): Psicología de las minorías activas, Madrid: Morata, 1981.

MoscovicI, S.: «A propos des recherches sur l'influence des minorites», Simposium Internacional sobre Procesos de Influencia Social, Barcelona, septiembre 1980.

Moscovicr, S. et al.: «Influence of a Consistent Minority on the Responses of a Majority in a Color Perception Task», Sociometry, 1969, 32, 365-379.

MoscovicI, S., y NemeTh, C. J.: «Social Influence II: Minority Influence», en Nemeth, C. (ed)., Social Psychology: Classic and Contemporary Integrations; Chicago: Rand-McNally, 1974.

MugnY, G.: «Difusión minoritaria de una innovación», en Doise, W. et al. Psicologia Social Experimental, Barcelona: Hispano Europea, 1980.

MuGNY, G.: «L'Influence du social dans l'influence social», Simposium Internacional sobre Procesos de Influencia Social, Barcelona, septiembre 1980. 
MugNY, G.: El «poder» de las minorias, Barcelona: Rol, 1981.

Mugny, G., y Papastamou, S.: «Cuando la rigidez persiste: la individualización y la psicologización como resistencias a la difusión de las innovaciones de la minoría», Clínica y Análisis Grupal, 1980, 5, 610-630.

Mugny, G., y Papastamou, S.: «Niveles de análisis en el estudio experimental de los procesos de influencia social», Clínica y Análisis Grupal, 1980, 5, 706-746.

Nemeth, C. J.: "Conceptuals Dinstinctions in Minority Influence», Simposium Internacional sobre Procesos de Influencia Social, Barcelona, septiembre 1980.

Nemetr, C. J.: «Stability of Faction Position and Influence», en Brandstatter, H. et al. (eds.), Group Decision Making, Londres: Academic, 1982.

Nemeth, C. J., y W A Necessary Condition for Minority Influence», Sociometry, 1974, 37, 529-540.

Nemeth, C. J. et al.: «Patterning of the Minority's Responses and their Influence en the Majority", European J. of Soc. Psychol., 1974, 4, 53-64.

Nemeth, C. J. et al.: «Increasing the Size of the Minority: Some Gains and some Losses», European J. of Soc. Psychol., 1977, 7, 15-27.

Nord, W. R.: «Social Exchange Theory: An Integrative Approach to Social Conformity», Psychol. Bull., 1969, 3, 174-208.

Personnaz, B.: «Niveau de résistance a l'influence de résponses nomiques et anomiques. Etude des phénomenes de référents clandestins et de conversionn, Recherches de psychologie sociale, $1979,1$.

Ross, L. et al:: «The Role of Attribution Processes in Conformity and Dissent: Revisiting the Asch Situation», American Psychologist, 1976, 31, 148-157.

SHeRIf, M. (1936): The Psychology of Social Norms, Nueva York: Harper and Row, 1966.

Thibaut, J. W., y Kelley, H. H.: The Social Psychology of Groups, Nueva York: Wiley, 1959.

WILDER, D. A.: "Perception of Groups, Size of Opposition, and Social Influence», J. of Experimental Soc. Psychol., 1977, 13, 253-268.

Willis, R. H.: «Conformity, Independence and Anticonformity», Human Relat., 1965, 18, 373-388. 